\title{
ORIGINAL ARTICLE \\ Inbreeding within human Schistosoma mansoni: do host-specific factors shape the genetic composition of parasite populations?
}

\author{
F Van den Broeck ${ }^{1,2}$, L Meurs ${ }^{2}$, JAM Raeymaekers ${ }^{3}, \mathrm{~N}_{\text {Boon }}{ }^{1,2}$, TN Dieye ${ }^{4}$, FAM Volckaert ${ }^{1}$, \\ K Polman ${ }^{2}$ and T Huyse ${ }^{1,2,5}$
}

The size, structure and distribution of host populations are key determinants of the genetic composition of parasite populations. Despite the evolutionary and epidemiological merits, there has been little consideration of how host heterogeneities affect the evolutionary trajectories of parasite populations. We assessed the genetic composition of natural populations of the parasite Schistosoma mansoni in northern Senegal. A total of 1346 parasites were collected from 14 snail and 57 human hosts within three villages and individually genotyped using nine microsatellite markers. Human host demographic parameters (age, gender and village of residence) and co-infection with Schistosoma haematobium were documented, and S. mansoni infection intensities were quantified. F-statistics and clustering analyses revealed a random distribution (panmixia) of parasite genetic variation among villages and hosts, confirming the concept of human hosts as 'genetic mixing bowls' for schistosomes. Host gender and village of residence did not show any association with parasite genetics. Host age, however, was significantly correlated with parasite inbreeding and heterozygosity, with children being more infected by related parasites than adults. The patterns may be explained by (1) genotype-dependent 'concomitant immunity' that leads to selective recruitment of genetically unrelated worms with host age, and/or (2) the 'genetic mixing bowl' hypothesis, where older hosts have been exposed to a wider variety of parasite strains than children. The present study suggests that host-specific factors may shape the genetic composition of schistosome populations, revealing important insights into host-parasite interactions within a natural system. Heredity (2014) 113, 32-41; doi:10.1038/hdy.2014.13; published online 12 March 2014

\section{INTRODUCTION}

As parasites and their hosts are closely associated, Price (1980) predicted that parasite infrapopulations (that is, all parasites of the same species within an individual host; Bush et al., 1997) would represent isolated patches, resulting in low genetic diversity and high levels of inbreeding. This seemed particularly true for recurrent generations of parasites infecting a single host (for example, phytophagous insects; Price, 1980). However, the majority of animal macroparasites (for example, flatworms, nematodes) release their offspring into the environment or multiply within one or more intermediate host species, mostly resulting in high genetic diversity levels similar to those reported in free-living organisms (Bush et al., 2001). It has been suggested that the high genetic diversity of macroparasites within individual hosts may reflect the tendency of these hosts to sample a number of transmission sites (Anderson et al., 1995; Nadler, 1995), thereby becoming 'genetic mixing bowls' for parasite genes (Curtis and Minchella, 2000; Curtis et al., 2002). The result is that parasite offspring are well mixed in the environment and that parasite genetic variation is randomly distributed between hosts (Criscione et al., 2005). As emphasized by Nadler (1995), many factors contribute to patterns of genetic diversity in parasites, such as the mating system (selfing, clonal, outcrossing), the number of intermediate host species and whether parasites transmit on land, in the water or both (for example, Criscione and Blouin, 2004). Studying the factors that affect the distribution of parasite genotypes among infrapopulations represents a powerful tool for understanding transmission dynamics. The joint analysis of the population genetic structure of the parasite with characteristics of the host such as age, gender and mobility can generate important insights in parasite evolution (factors that shape local adaptation and speciation) as well as epidemiology (determining whether transmission is focal or not; Criscione, 2008).

The digenean trematode Schistosoma mansoni has a complex twohost life cycle with an asexual amplification stage in the snail intermediate host, yielding thousands of clonal cercariae that infect the human final host. The cercariae develop into dioecious worms that reproduce sexually, resulting in offspring (miracidia) upon hatching of worm eggs that are released into the external environment through feces. Population genetic studies have shown that the geographic scale of differentiation of $S$. mansoni varies substantially between study areas. For example, in Melquiades (Brazil), a village with a complex hydrosystem of ridges and valleys, high differentiation

${ }^{1}$ Laboratory of Biodiversity and Evolutionary Genomics, University of Leuven, Leuven, Belgium; ${ }^{2}$ Unit of Medical Helminthology, Institute of Tropical Medicine, Antwerp, Belgium; ${ }^{3}$ Zoological Institute, University of Basel, Basel, Switzerland; ${ }^{4}$ Laboratory of Bacteriology and Virology, Aristide Le Dantec University Hospital, Dakar, Senegal and ${ }^{5}$ Section Invertebrates, Royal Museum for Central Africa, Tervuren, Belgium

Correspondence: F Van den Broeck, Laboratory of Biodiversity and Evolutionary Genomics, University of Leuven, Ch. Deberiotstraat 32, Leuven B-3000, Belgium. E-mail: fvandenbroeck@gmail.com

Received 23 August 2013; revised 9 January 2014; accepted 17 January 2014; published online 12 March 2014 
was found between $S$. mansoni populations from different households along different water bodies (Thiele et al., 2008). Low differentiation was found in Virgem das Gracas (Brazil) where households were situated along the same water body (Thiele et al., 2008). The authors argued that the complex geography of Melquiades probably restricted host movement for water usage, resulting in focal points of transmission and thus high genetic differentiation. These data suggest that the gene flow of $S$. mansoni may be determined by human host mobility and that gene flow may occur across large geographical distances in the absence of boundaries between transmission sites (Steinauer et al., 2010). Similar conclusions were drawn from studies in Kenya (Agola et al., 2009) and for non-human foci (Rattus rattus) of S. mansoni (Sire et al., 2001; Prugnolle et al., 2005a).

Apart from host movement for water usage (influenced by geographical entities), other host-specific factors could, directly or indirectly, affect the distribution of $S$. mansoni strains within and among hosts. For instance, the genetic diversity of $S$. mansoni infrapopulation within male $R$. rattus hosts was higher than within female hosts (Caillaud et al., 2006). The authors explained this by differences in immunocompetence or water contact behavior between male and female rat hosts. Similar explanations (resistance and/or exposure to infection) were put forward to explain the ageand gender-dependent infection intensities observed within human schistosomiasis endemic communities (Gryseels, 1994; Kabatereine et al., 1999). Nevertheless, studies on the impact of host-specific factors on the genetic structure of schistosome populations are scarce (Thiele et al., 2008; Gower et al., 2011). This is mainly due to the methodological limitations of collecting and genotyping larval field stages. So far, only one study on human S. mansoni infections incorporated host-specific factors (gender and age) in their parasite genetic analyses (Thiele et al., 2008). No relationship between parasite population structure and host-specific factors was detected (Thiele et al., 2008), possibly because $S$. mansoni genotypes were inferred after laboratory passage in mice. This strategy most likely induced a bias due to genetic bottlenecking or differential immune responses of laboratory animals.

In this study, we investigated host-specific factors that may shape the genetic composition of natural S. mansoni parasite populations in northern Senegal. In total, 1346 parasites were collected from 14 snail and 57 human hosts from three villages. By genotyping larval schistosomes directly isolated from humans, the need for laboratory passage in mice was avoided. This allows an improved analysis of the interaction between human host-specific factors and schistosome population genetics.

\section{MATERIAL AND METHODS}

\section{Ethical statement}

This study is part of a larger investigation of the epidemiology, transmission and control of schistosomiasis in Senegal (SCHISTOINIR: www.york.ac.uk/res/ schistoinir). Ethical approval was obtained from 'Le Comité National d'Ethique de la Recherche en Santé' in Senegal, the review board of the Institute of Tropical Medicine in Belgium and the ethical committee of the Antwerp University Hospital in Belgium. Informed and written consent was obtained from all participants before the start of the study. All inhabitants were treated after the study according to WHO guidelines (WHO, 2006) with a single dose of praziquantel $(40 \mathrm{mg} / \mathrm{kg}$ of body weight $)$ and mebendazole (500 mg) for schistosomiasis and soil-transmitted helminthiasis, respectively.

\section{Description of the study sites and epidemiology}

Parasites and host demographic data were obtained from the villages Pakh $\left(16^{\circ} 24^{\prime} 12^{\prime \prime} \mathrm{N} 15^{\circ} 48^{\prime} 42^{\prime \prime} \mathrm{W} ; 790\right.$ inhabitants), Diokhor Tack $\left(16^{\circ} 11^{\prime} 24^{\prime \prime} \mathrm{N}\right.$ $15^{\circ} 52^{\prime} 48^{\prime \prime} \mathrm{W}$; 984 inhabitants) and Ndieumeul (also known as Thiekène; $16^{\circ} 13^{\prime} 12^{\prime \prime} \mathrm{N} 15^{\circ} 51^{\prime} 36^{\prime \prime} \mathrm{W} ; 384$ inhabitants), all located on the western side of Lake Guiers (northern Senegal). Diokhor Tack and Ndieumeul lie in close proximity $(4 \mathrm{~km})$ on the Peninsula Nouk Pomo, situated about $22 \mathrm{~km}$ from Pakh that lies next to a large irrigation canal close to the lake (Figure 1). Almost all inhabitants (99\%) in Ndieumeul and Diokhor belong to the ethnic group Wolof while in Pakh they belong to Wolof (63\%) and Peul (30\%). To our knowledge, there have been no anthelminthic treatment programs in these villages before the study.

The prevalences and intensities of S. mansoni infection increased up to the second decade of life, with a subsequent decrease in adults (Meurs et al., 2012). S. mansoni prevalences were $16 \%$ in Pakh, $75 \%$ in Ndieumeul and $55 \%$ in Diokhor. Schistosoma haematobium was co-endemic in all the villages $57 \%$ in Pakh, 66\% in Ndieumeul and $44 \%$ in Diokhor) with mixed infection prevalences of $12 \%$ in Pakh, $55 \%$ in Ndieumeul and 32\% in Diokhor (Meurs et al., 2012; unpublished data).

\section{Data collection from humans and snails}

In total, three surveys were conducted within less than a year during February 2009 (Pakh), August 2009 (Pakh, Diokhor Tack and Ndieumeul) and January 2010 (Ndieumeul). Stool samples were collected from all participants and processed by the Kato-Katz technique as described elsewhere (Meurs et al., 2012). S. mansoni infection intensity was expressed as the number of eggs detected per gram of feces (EPG). From each participant, data were collected on age, gender and village of residence. S. mansoni eggs were filtered from positive stool samples by homogenizing each sample with water and then passing it through a metal sieve of $212 \mu \mathrm{m}$ pore size to remove any larger debris. Eggs were then concentrated in Petri dishes by passing the remaining aqueous solution through a homemade Pitchford and Visser funnel. Eggs were either pooled per inhabitant in $1.5 \mathrm{ml}$ tubes filled with $\mathrm{EtOH}(70 \%)$ or hatched for miracidia that were individually pipetted onto Whatman FTA indicator cards (GE Healthcare Bio-Sciences AB, Uppsala, Sweden) in a volume of $3 \mu \mathrm{l}$ of water.

Snails of the species Biomphalaria pfeifferi were collected within a 2-year period during August 2008 (Mbane, $16^{\circ} 16^{\prime} 15^{\prime \prime} \mathrm{N} 15^{\circ} 48^{\prime} 07^{\prime \prime} \mathrm{W}$; Temey, $16^{\circ} 19^{\prime} 45^{\prime \prime} \mathrm{N} \quad 15^{\circ} 46^{\prime} 04^{\prime \prime} \mathrm{W}$; Nder, $16^{\circ} 16^{\prime} 00^{\prime \prime} \mathrm{N} 15^{\circ} 52^{\prime} 28^{\prime \prime} \mathrm{W}$; Yetti Yone, $16^{\circ} 20^{\prime} 56^{\prime \prime} \mathrm{N} 15^{\circ} 53^{\prime} 4^{\prime \prime} \mathrm{W}$; Roumbatine, $16^{\circ} 17^{\prime} 19^{\prime \prime} \mathrm{N} 15^{\circ} 52^{\prime} 58^{\prime \prime} \mathrm{W}$ and Pakh), February 2009 (Pakh), August 2009 (Diokhor, Ndieumeul and Pakh) and January 2010 (Ndieumeul and Pakh; Figure 1). Not all transmission sites could be sampled at all times as some dried out or were inaccessible due to vegetation or inundation (see Supplementary Table S1 for details on which sites were sampled). Each transmission site was thoroughly sampled by two researchers for at least $15 \mathrm{~min}$ and longer when many snails were found. Snails were stimulated to shed cercariae by transferring them in containers with bottled water and exposing them for 5-10 min to direct sunlight. Cercariae were individually fixated in 96-well plates containing $40 \mu \mathrm{EtOH}$ (70\%). All samples were stored and transported at room temperature.

\section{Molecular analysis}

Genomic DNA extraction and genotyping was performed as described before (Van den Broeck et al., 2011). In short, individual S. mansoni parasites were genotyped using nine putatively neutral microsatellite markers (L46951, CA111, S9-1, SMD11, SMD25, SMD28, SMD43, SMD89, SMDA28; Durand et al., 2000; Blair et al., 2001; Curtis et al., 2001). PCR products were analyzed using an ABI 3130 Genetic Analyser (Applied Biosystems, Foster City, CA, USA) and GeneScan 500 LIZ (Applied Biosystems) as size standard. Allele sizes were manually verified using GENEMAPPER v4.0 (Applied Biosystems). Miracidia that were successfully genotyped for less than seven of the nine loci were excluded from the analyses. As more hosts, rather than more miracidia per host, leads to a more robust estimate of parasite population genetic diversity (French et al., 2012), all hosts were included for data analysis except those that were genotyped for only one parasite. For each infected snail host, eight cercariae were genotyped, unique multi-locus genotypes (MLGs) were identified and used for data analysis while the identical MLGs were removed.

\section{Genetic diversity}

For each village and for each host, parasite observed $\left(H_{0}\right)$ and Nei's unbiased expected heterozygosity $\left(H_{\mathrm{s}}\right)$ were calculated using GENETIX v4.05 


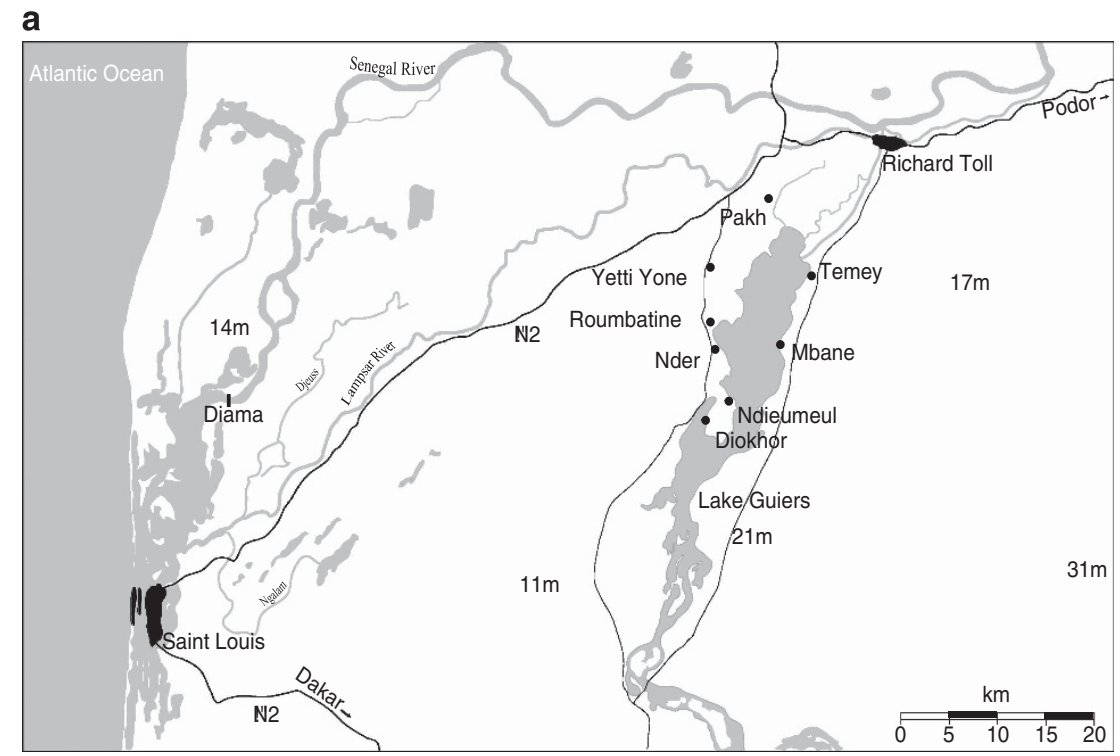

b

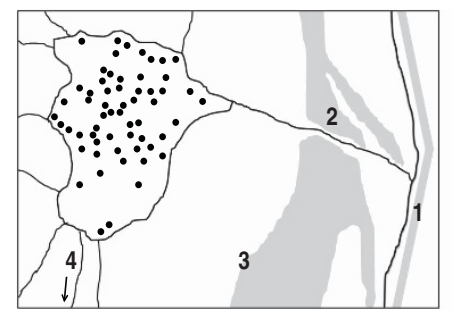

c

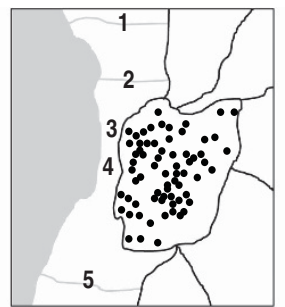

d

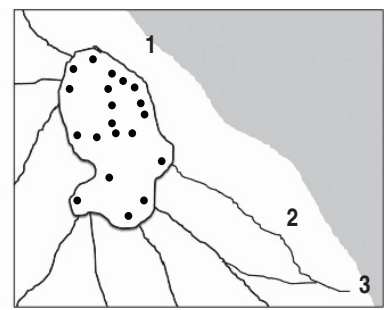

Figure 1 (a) Map of northern Senegal showing the three major water bodies (Lake Guiers, Senegal River and its tributaries Lampsar River and Djeuss), the national road N2, cities Saint-Louis and Richard Toll and the location of the study villages (Pakh, Yetti Yone, Roumbatine, Nder, Diokhor Tack, Ndieumeul, Mbane and Temey). While snails were collected in all villages, human samples were only collected in Pakh, Diokhor and Ndieumeul. The elevation of some geographic points are indicated in meters above sea level. Detailed maps of Pakh (b), Diokhor (c) and Ndieumeul (d): dots are households, black lines are 'roads', grey areas are water and numbers indicate the known transmission sites within each village.

(Belkhir et al., 1996-2004). The inbreeding coefficient $F_{\mathrm{IS}}$, defined as the probability that two alleles at a locus are identical by descent, was estimated in GENETIX at the village and at the host level using $f$ (Weir and Cockerham, 1984), tested for significance using 10000 permutations and corrected for multiple testing using sequential Bonferroni corrections. Parasite allelic richness (AR; which corrects the number of alleles per locus for unequal sample sizes) was estimated based on a minimum of two alleles per host and per village using the R package HIERFSTAT v0.04-6 (Goudet, 2005). The same analyses were also performed for the cercarial population of Ndieumeul, which was the only sample with a sufficient amount of infected snails (see Results section).

\section{Temporal and spatial genetic structure}

To assess the relative importance of the temporal to the spatial genetic variation, differences in allele frequencies were sought between sampling times within one village. To this end, the function varcomp.glob as implemented in the $\mathrm{R}$ package HIERFSTAT was used to test whether alleles were correlated within one sampling time relative to the total observed variation within each village independently (for Ndieumeul and Pakh, as Diokhor Tack was surveyed only once). This nested design was suggested as a solution for crossed factors (that is, different villages were sampled at the same sampling time; de Meeus and Goudet, 2007). Significance was tested by 10000 permutations of host samples among sampling times in each village independently (function test.between).

Parasite gene flow within and between hosts and villages was assessed using a hierarchical analysis of genetic structure that quantified the subdivision of parasites ('Par') at two levels: within hosts ('Pop') and within villages ('Vil').
The terminology for the classical F-statistics was modified as follows: $F_{\mathrm{Vi} / / T o t a l}$ reflects the correlation of alleles within villages relative to the total; $F_{\text {Pop/Vil }}$ reflects the correlation of alleles within hosts relative to the village, and $F_{\mathrm{Par} / \mathrm{Pop}}$ reflects the correlation of alleles within parasites relative to the host. Each of these hierarchical estimates was computed using the R package HIERFSTAT, both overall (function varcomp.glob) as for each locus separately (function varcomp). Overall $F_{\mathrm{ST}}$ estimates were standardized by dividing the observed $F_{\mathrm{ST}}$ values calculated from the data by the maximum $F_{\mathrm{ST}}$ value given the data (Hedrick, 2005) using RecodeData v.0.1 (Meirmans, 2006). Significance of all $F$-values was tested with 10000 permutations. Using FSTAT v2.9.3 (Goudet, 2005), alleles were permuted among parasites within hosts $\left(F_{\mathrm{Par} / \mathrm{Pop}}\right)$ while HIERFSTAT was used for testing the significance of $F_{\text {Pop/Vil }}$ (parasites were permuted among hosts but kept within their village of origin; function test.within) and of $F_{\mathrm{Vil} / \mathrm{Total}}$ (whole host samples were permuted among villages; function test.between). All $P$-values were corrected for multiple testing. Finally, 10000 bootstraps were performed over loci in HIERFSTAT to obtain confidence intervals (CIs) for every F-statistic (function boot.vc).

Population structure was further analyzed with a Bayesian Markov chain Monte Carlo (MCMC) clustering analysis as implemented in STRUCTURE v2.2.3 (Pritchard et al., 2000). This method assigns individuals probabilistically to $K$ populations or jointly to two or more populations if their genotypes indicate they are admixed, in such a way that loci within populations are in Hardy-Weinberg and linkage equilibrium. The number of clusters $K$ was derived assuming the admixture model and correlated allele frequencies; three replicate runs were run for each predefined $K$ (ranging from 1 to 10). Each run was initiated by 100000 burn-in steps and consisted of 1000000 MCMC steps. 
The optimal $K$ value was identified by the highest $\ln \mathrm{P}(\mathrm{D})$ value and by estimating $\Delta K$, which is based on the second-order rate change of the posterior $\ln \mathrm{P}(\mathrm{D})$ (Evanno et al., 2005).

\section{Impact of Wahlund effect, inbreeding and biased sampling on variations in $F_{\text {IS }}$}

F-statistics revealed variable levels of parasite $F_{I S}$ estimates at the host level, ranging from some negative (heterozygote excess) to many positive deviations (heterozygote deficiency) from Hardy-Weinberg equilibrium (HWE; see Results section). Excluding the possibility of scoring errors (see Van den Broeck et al., 2011), a more in-depth analysis of three competing mechanisms related to the biology of schistosomes were performed following a similar approach as presented by Castric et al. (2002).

First, heterozygote deficiencies could result from a subdivision of the local population into isolated and differentiated reproductive units. When hosts are infected with parasites from spatially or temporally separated gene pools (that is, cryptic population structure), fewer heterozygotes are expected than under random mating, resulting in a Wahlund effect. Tests for Wahlund effects were performed using STRUCTURE, as described above, which is a method that aims at unraveling cryptic population structure by assigning individuals to $K$ populations as such that the populations are in Hardy-Weinberg and linkage equilibrium. When the optimal $K$ value was $\geqslant 2$, then this means the presence of population structure, which could possibly lead to a Wahlund effect.

A second cause for heterozygote deficiencies is mating between close relatives, that is, inbreeding. When paired adult worms are genetically related, the chance that two alleles in the offspring are identical by descent will be higher than expected under random mating. Inbred offspring will be homozygous for most loci and will therefore show lower individual heterozygosity than offspring resulting from random mating. We therefore estimated the proportion of heterozygous loci per parasite (that is, multi-locus heterozygosity (MLH)) and compared the mean observed MLH per host $\left(\mathrm{MLH}_{\mathrm{obs}}\right)$ with the expected under random mating $\left(\mathrm{MLH}_{\mathrm{exp}}\right)$. The expected distribution was obtained from 1000 pseudosamples in which alleles were randomly associated within individuals using GENETIX. Significance was tested by estimating $P$-values as the probability of observing lower MLH than expected under random mating. To test whether deviations in HWE were due to inbreeding, an association was sought between $F_{\text {IS }}$ and individual heterozygote deficiency as estimated by $\left(\mathrm{MLH}_{\mathrm{obs}}-\mathrm{MLH}_{\mathrm{exp}}\right) / \mathrm{MLH}_{\mathrm{exp}}$.

A final mechanism that could explain variations in $F_{\text {IS }}$ is the non-random sampling of offspring from a limited number of families. As adult worms reside in the human blood vessels, genetic analyses could only be performed on offspring genotypes without having an idea about the number of adult worms that contributed to this pool of offspring (Steinauer et al., 2013). As some hosts might harbor less adult worms than others, the probability of sampling two related offspring parasites is higher than expected in a randomly mating population. Such a non-random sampling of offspring from a limited number of families will result in heterozygote excesses or at least a downward biased estimation of $F_{\text {IS. }}$. We therefore assessed the genetic relatedness of every pair of offspring within a sample and tested whether the mean observed relatedness was higher than expected. Pairwise relatedness between each individual parasite was estimated according to the identity coefficient as it has a smaller variance than other estimates of relatedness (Belkhir et al., 2002). The expected distributions were generated by 1000 permutations of genotypes in IDENTIX v1.1 (Belkhir et al., 2002), and significance was tested by estimating $P$-values as the probability of observing higher relatedness than expected.

\section{Link between human host-specific factors and $S$. mansoni parasite genetics}

Associations between parasite population genetic statistics $\left(H_{\mathrm{o}}, H_{\mathrm{s}}, \mathrm{AR}\right.$ and $\left.F_{\mathrm{IS}}\right)$ and human host demographic variables (age, gender and village of residence), infection intensity (EPG) and co-infection with S. haematobium were analyzed using Statistica v11 (StatSoft, Tulsa, OK, USA). A general linear model was constructed for each parasite population statistic in order to test their dependency on individual host demographic parameters, infection intensity and co-infection with S. haematobium. Host age and infection intensity were included in the model as continuous covariates and host gender (male, female), co-infection (absent, present) and village of residence (Pakh, Diokhor Tack and Ndieumeul) as categorical variables. All interactions were tested but excluded from the model when not significant.

Two additional tests were finally performed to investigate the robustness of the statistical outcome. First, to test the effect of low sample sizes, hosts that were genotyped for $<13$ parasites were removed, resulting in a data set comprising only 45 human hosts. The same general linear models as described above were then repeated. Second, the effect of individual loci was assessed following a jack-knife approach: each locus was individually removed, parasite population statistics were re-estimated using the remainder of the loci and univariate statistics were then performed for each jack-knifed estimate.

\section{RESULTS}

\section{Data set}

A total of 2891 S. mansoni parasites from 63 hosts were subjected to genotyping, which is on average 45.9 parasites per host $(\mathrm{min} .=2$, max. $=157$, median $=46$ ). Of these, 1345 genotypes (that is, $46.5 \%$ ) were successfully scored for at least seven out of nine loci and were used for further analyses. The failure rate depended heavily on the type of sample: eggs were genotyped with an average success of $44.2 \%$ while miracidia showed almost double the success rate (that is, $81.2 \%$ ). The final data set consisted of 57 human hosts of which 37 were female and 23 were male hosts; 12 hosts originated from the village Diokhor Tack, 11 from Pakh and 37 from Ndieumeul (Table 1). Host age ranged between 4 and 50 years and differed significantly between the three villages (one-way analysis of vriance; $F=8.02 ; P=0.0009$ ), in particular because of the young age of the Ndieumeul sample (mean age was 23 years in Diokhor Tack, 29 years in Pakh and 14 years in Ndieumeul). Each host harbored on average 24 parasites $(\min .=2$, $\max .=74$; Table 1$)$. All parasites presented unique MLGs.

In total, $283 B$. pfeifferi snails were collected in eight villages (Supplementary Table S1). Infected snails were only found in three villages: in Temey and Nder during August 2008 and in Ndieumeul during January 2010. Snail S. mansoni prevalences were 3.5\% (1/29) in Nder, 9.62\% (13/135) in Ndieumeul and $14.3 \%$ in Temey (1/7). Cercarial MLGs were successfully obtained from all snails, except for one snail from Ndieumeul. Two snails from Ndieumeul were each infected with two different $S$. mansoni strains (that is, two different MLGs) while all the other snails were infected with one parasite strain only, resulting in a final cercarial population of 14 MLGs in Ndieumeul. In Nder and Temey, only one MLG per infected snail was found. No snail harbored near-identical genotypes (that is, genotypes that differed at one or a very few loci from a frequently observed MLG).

\section{Genetic diversity}

All measures of genetic diversity were relatively uniform among the different sampling sites, although S. mansoni populations from Pakh $\left(\mathrm{AR}=1.55 ; H_{\mathrm{s}}=0.55\right)$ were slightly more diverse than those from Diokhor Tack $\left(\mathrm{AR}=1.54 ; H_{\mathrm{s}}=0.54\right)$ and Ndieumeul $(\mathrm{AR}=1.53$; $H_{\mathrm{s}}=0.53$; Table 1$)$. The genetic diversity of the cercarial population in Ndieumeul $\left(\mathrm{AR}=1.57 ; H_{\mathrm{s}}=0.49\right)$ was similar to the parasite genetic diversity found within each village and each host (Table 1).

\section{Temporal and spatial genetic structure}

The genetic variation of $S$. mansoni within the three communities showed no spatial or temporal structure. The variance explained by the factor time in both Pakh $\left(F_{\text {SamplingTime } / \text { Pakh }}=0.001\right.$; CI: -0.004 to $0.005 ; P=0.177)$ and Ndieumeul $\left(F_{\text {SamplingTime } / \text { Ndieumeul }}=0.008 ; \mathrm{CI}\right.$ : -0.001 to $0.025 ; P=0.088$ ) was low and insignificant. Although 
Table 1 Genetic diversity of $S$. mansoni populations as estimated independently per host and per village

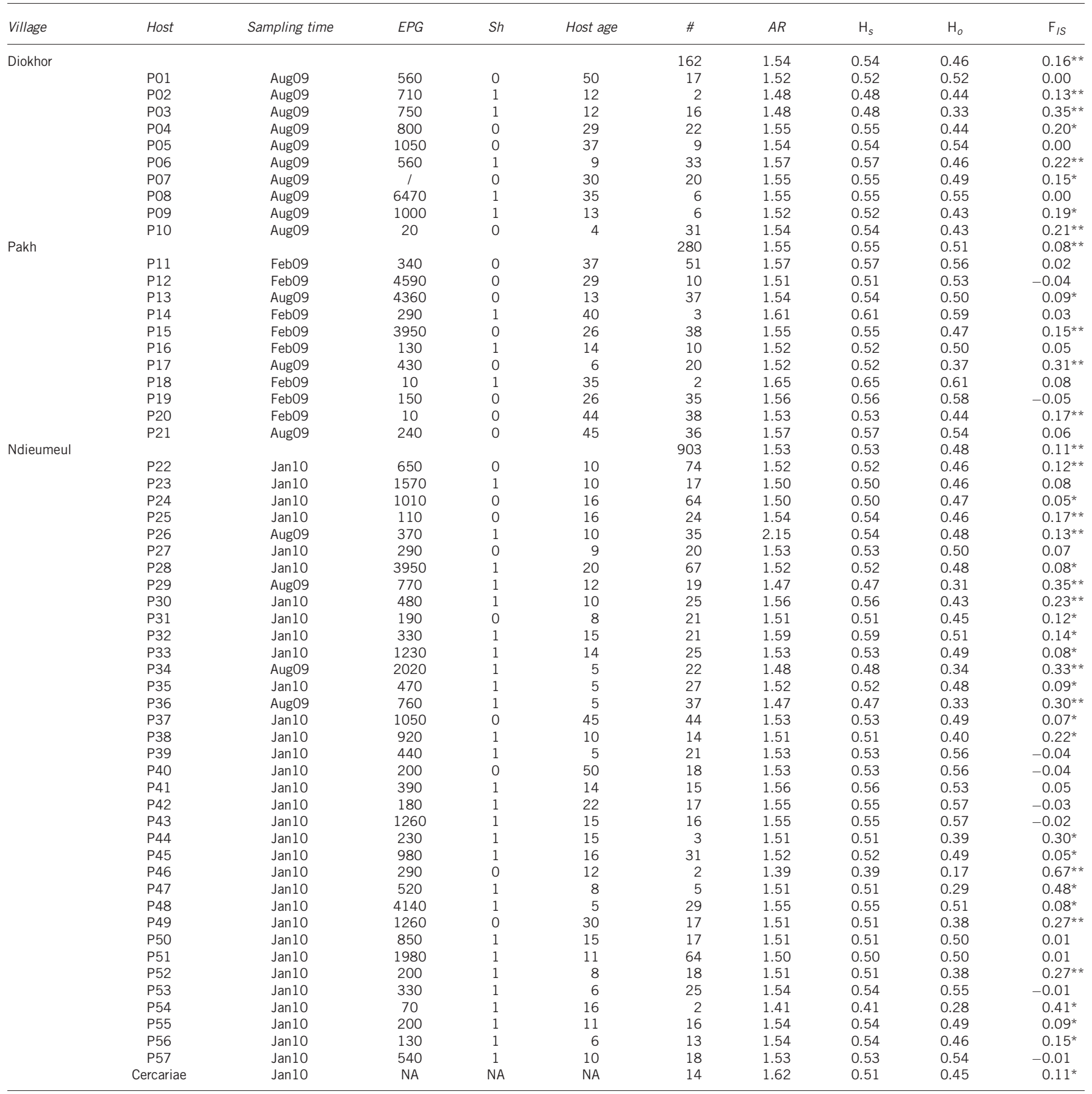

Abbreviations: AR, allelic richness; EPG, eggs per gram of feces; $F_{\mathrm{IS}}$, inbreeding coefficient; $H_{0}$, observed heterozygosity; $H_{\mathrm{s}}$, unbiased expected heterozygosity; NA, not applicable; Sh, co-infection with $S$. haematobium; \#, number of successfully genotyped parasites.

Significant $F_{\mathrm{IS}}$ values were indicated with * when $P<0.05$ and ${ }^{* *}$ when $P<0.001$ (that is, Bonferroni corrected). The information on gender of the host was excluded from this table to preserve anonymity.

significant, the estimates of spatial differentiation among villages relative to the whole sampling area were very low $\left(F_{\mathrm{Vil} / \mathrm{Total}}=-0.003\right.$; CI: $0.002-0.004 ; P<0.001$; Table 2). Similar results were obtained at the host level $\left(F_{\text {Pop/Vil }}=0.004 ;\right.$ CI: $0.002-0.006 ; \quad P<0.001$; Table 2).

Hierarchical analyses showed that alleles were highly correlated within parasites relative to the host $\left(F_{\mathrm{Par} / \mathrm{Pop}}=0.102\right.$; CI: $0.074-0.135$; $P<0.001$; Table 2), which were on average a hundred times higher than the estimates at the host and the village level (Table 2). This result was confirmed when host samples were considered independently: about 48 hosts harbored parasite populations showing positive $F_{\text {IS }}$ values, from which 27 were highly significant $(P<0.01$; Table 1$)$. Only eight hosts harbored parasite populations showing negative $F_{I S}$ values; none of them were significant.

Analysis in STRUCTURE v2.2.3 revealed no subdivision of populations as no optimal $K$ value could be identified; the highest $\ln \mathrm{P}(\mathrm{D})$ value was obtained for $K=1$ (Supplementary Figure S1). The membership probabilities ( $Q$-values) for $K=2$ and higher were 
uniform across clusters (Supplementary Figure S1), indicating that individuals were inconclusively assigned to the clusters, which furthermore supports the results that $K=1$.

\section{Evidence for inbreeding}

Both the Wahlund effect and biased sampling were rejected as systematic explanations for departures of HWE at the host level. STRUCTURE analyses revealed a homogenized population, thereby suggesting a minimum impact of the Wahlund effect (Supplementary Figure S1). Mean pairwise relatedness between parasites from each host did not depart significantly from its expected distribution for almost all host samples (Figure 2; see Supplementary Table S2 for exact $P$-values). Only host P46 potentially suffered from biased sampling and was therefore removed from subsequent statistical analyses (Figure 2). On the contrary, evidence was found of a shift to lower individual heterozygosity (MLH) than expected under random mating (Figure 2; see Supplementary Table S2 for exact $P$-values) for many hosts (33), and variations in $F_{I S}$ were strongly associated with variations in individual heterozygote deficiencies $\left(\mathrm{MLH}_{\mathrm{obs}}-\right.$ $\mathrm{MLH}_{\text {exp }}$ ) $/ \mathrm{MLH}_{\text {exp }}$ (Pearson's correlation test, $P<0.001 ; r=-0.97$ ). Altogether, these results suggest that the variation observed in $F_{\mathrm{IS}}$ estimates between hosts is most likely due to variations in levels of parasite inbreeding.

Table 2 Hierarchical analysis of parasite genetic diversity overall and per locus at three levels: within villages $\left(F_{\mathrm{Vil} / \text { Total }}\right)$, within hosts $\left(F_{\mathrm{Pop} / \mathrm{Vil}}\right)$ and within individual parasites $\left(F_{\mathrm{Par} / \mathrm{Pop}}\right)$

\begin{tabular}{|c|c|c|c|c|}
\hline Marker & \# Alleles & $\mathrm{F}_{\text {Vil/Total }}$ & $\mathrm{F}_{\text {Pop/Vil }}$ & $\mathrm{F}_{P a r / P o p}$ \\
\hline$L 46951$ & 7 & 0.002 & $0.006 *$ & $0.078 * *$ \\
\hline CA11-1 & 7 & $0.003^{*}$ & -0.001 & $0.085^{* *}$ \\
\hline S9-1 & 5 & -0.001 & 0.002 & $0.082^{* *}$ \\
\hline SMD11 & 31 & $0.002^{* *}$ & $0.007 * *$ & $0.109 * *$ \\
\hline SMD25 & 8 & $0.005^{*}$ & $0.004^{*}$ & $0.090^{* *}$ \\
\hline SMD28 & 5 & 0.002 & $0.008^{*}$ & 0.015 \\
\hline SMD43 & 15 & $0.003^{*}$ & $0.004 * *$ & $0.179 * *$ \\
\hline SMD89 & 5 & 0.006 & -0.004 & $0.059 *$ \\
\hline SMDA28 & 11 & $0.004^{* *}$ & 0.001 & $0.067^{* *}$ \\
\hline Overall (Cl: 2.5-97.5\%) & 10,4 & $0.003^{* *}(0.002-0.004)$ & $0.004 * *(0.002-0.006)$ & $0.102 * *(0.074-0.135)$ \\
\hline Overall (standardized) & NA & 0.007 & 0.009 & NA \\
\hline
\end{tabular}

Abbreviations: $\mathrm{Cl}$, confidence interval; NA, not applicable.

Standardized estimates of genetic differentiation are given for $F_{\mathrm{V} i \text { ivtal }}$ and $F_{\mathrm{Pop}}$ il after recoding the data following Meirmans (2006). Note that recoding the data does not change within population diversities; the estimate of $F_{\text {Par/Pop }}$ therefore remains the same. Number of alleles (\# Alleles) are given per locus and overall. Significance was indicated with * when $P<0.05$ and

** when $P<0.002$ (that is, Bonferroni corrected).
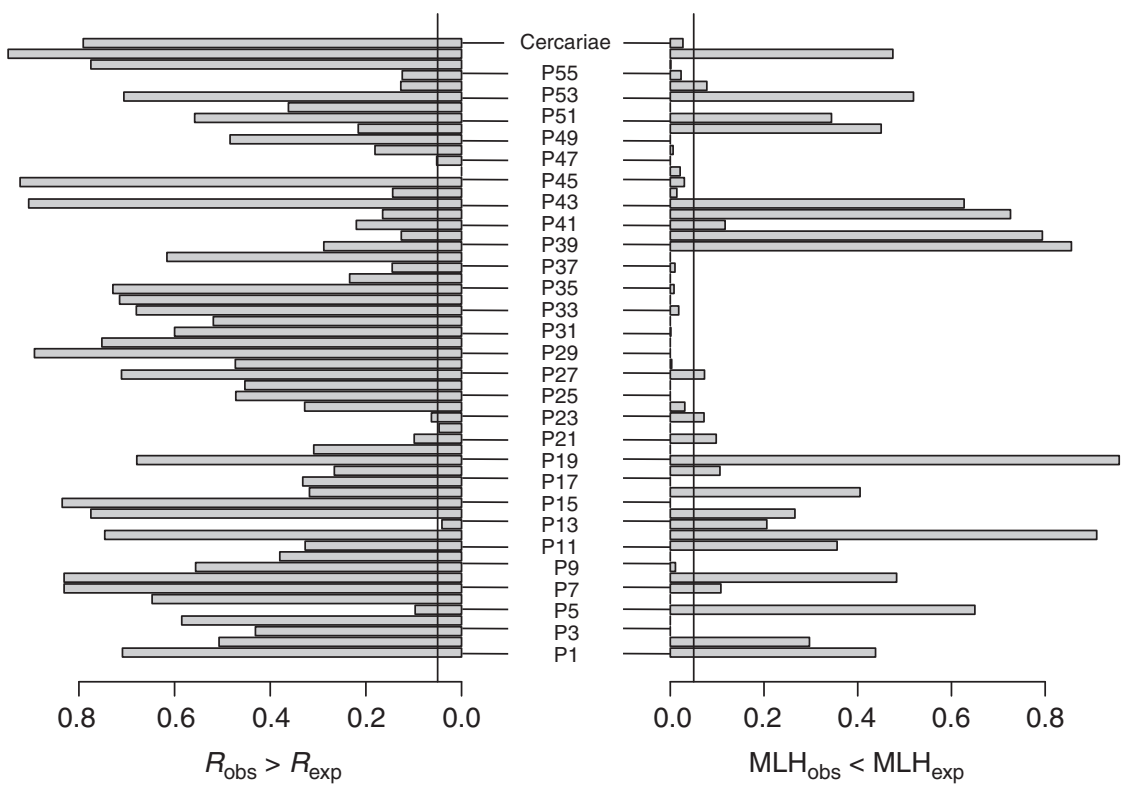

Figure $2 P$-values for parasite relatedness $(R)$ and multilocus heterozygosity (MLH) after 1000 randomizations. Each bar represents an individual human host (labeled according to the numbering in Table 1) or the pool of cercariae from the village Ndieumeul (named 'Cercariae'). Vertical lines were drawn for $P=0.05$; values smaller than this line are considered significant. $R_{\text {obs }}>R_{\text {exp }}$ reflects the probability of observing higher pairwise relatedness than expected, suggesting biased sampling of (many) siblings from a limited number of adults for this host. Only host 46 was highly significant for this test. $\mathrm{MLH}_{\mathrm{obs}}<\mathrm{MLH}_{\exp }$ reflects the probability of observing a lower individual heterozygosity than expected from random mating, suggesting parasite inbreeding. This test was significant for 33 human hosts. See Supplementary Table S2 for exact $P$-values. 
Link between human host-specific factors and $S$. mansoni parasite genetics

Model building revealed that host age was the only predictor explaining the variance in some parasite population statistics (Table 3). In a general linear model with host age, gender, infection intensity (EPG) and co-infection with S. haematobium, S. mansoni inbreeding $\left(F_{\mathrm{IS}}\right)$ was negatively associated with age of the host $(\beta=-0.004, P=0.018)$, whereas a significant increase in observed heterozygosity with host age was found $(\beta=0.003$, $P=0.005)$. Host age explained $11 \%$ of the variation in parasite inbreeding (adjusted $R^{2}=0.112, P=0.007$ ) and $17 \%$ of the variation in observed heterozygosity (adjusted $R^{2}=0.169$,
$P=0.001)$. No significant interactions between age and other covariates were found (results not shown). Results were similar when general linear models were repeated excluding hosts that harbored $<13$ genotyped parasites (Table 3), and jack-knifing over loci revealed that all loci equally contributed to these patterns (Table 4). These results suggest that the observed associations did not suffer from sampling bias in terms of number of hosts, number of genotyped parasites and number of markers. Expected heterozygosity $\left(H_{\mathrm{s}}\right)$ was significantly higher in the village Pakh than in Ndieumeul $(\beta=0.020 ; P=0.024)$ while $H_{\mathrm{s}}$ increased borderline significantly with age of the host $(\beta=0.0005, P=0.072$; Table 3$)$. No significant associations with $H_{\mathrm{s}}$ were found, however, when excluding hosts

Table 3 Results from general linear models examining the association of parasite population statistics with host demographic variables, infection intensity (EPG) and co-infection with S. haematobium

\begin{tabular}{|c|c|c|c|c|c|c|}
\hline \multirow[t]{2}{*}{ Parasite } & \multirow[t]{2}{*}{ Host } & & \multicolumn{2}{|c|}{ Minimum 2 parasites } & \multicolumn{2}{|c|}{ Minimum 13 parasites } \\
\hline & & & Estimate $\beta(95 \%$ Cl) & P-value & Estimate $\beta(95 \% \mathrm{Cl})$ & P-value \\
\hline \multirow[t]{10}{*}{$F_{\mathrm{IS}}$} & & Intercept & 0.219 & & 0.219 & \\
\hline & Village & Ndieumeul & Ref. & & Ref. & \\
\hline & & Diokhor & $0.032(-0.030,0.093)$ & 0.308 & $0.058(-0.014,0.131)$ & 0.112 \\
\hline & & Pakh & $-0.013(-0.076,0.050)$ & 0.690 & $-0.009(-0.085,0.065)$ & 0.790 \\
\hline & Gender & Female & Ref. & & Ref. & \\
\hline & & Male & $-0.004(-0.039,0.031)$ & 0.806 & $-0.020(-0.057,0.016)$ & 0.261 \\
\hline & Co-infection & Present & Ref. & & Ref. & \\
\hline & & Absent & $0.001(-0.039,0.041)$ & 0.948 & $0.009(-0.036,0.055)$ & 0.679 \\
\hline & Age & & $-0.004(-0.007,-0.001)$ & $0.018^{*}$ & $-0.004(-0.007,-0.0008)$ & $0.016^{*}$ \\
\hline & EPG & & $-2.10^{-5}\left(-4.10^{-5}, 1.10^{-5}\right)$ & 0.207 & $-1.10^{-5}\left(-4.10^{-5}, 3.10^{-5}\right)$ & 0.689 \\
\hline \multirow[t]{10}{*}{$H_{0}$} & & Intercept & 0.416 & & 0.425 & \\
\hline & Village & Ndieumeul & Ref. & & Ref. & \\
\hline & & Diokhor & $-0.024(-0.061,0.014)$ & 0.208 & $-0.034(-0.079,0.011)$ & 0.132 \\
\hline & & Pakh & $0.026(-0.012,0.064)$ & 0.177 & $0.021(-0.026,0.067)$ & 0.370 \\
\hline & Gender & Female & Ref. & & Ref. & \\
\hline & & Male & $0.005(-0.016,0.026)$ & 0.626 & $0.012(-0.011,0.034)$ & 0.291 \\
\hline & Co-infection & Present & Ref. & & Ref. & \\
\hline & & Absent & $-0.009(-0.034,0.014)$ & 0.418 & $-0.009(-0.038,0.019)$ & 0.497 \\
\hline & Age & & $0.003(0.0008,0.005)$ & $0.005^{* *}$ & $0.002(0.0003,0.004)$ & $0.023 *$ \\
\hline & EPG & & $4.10^{-6}\left(-1.10^{-5}, 2.10^{-5}\right)$ & 0.552 & $0.000\left(-2.10^{-6}, 2.10^{-5}\right)$ & 0.971 \\
\hline \multirow{10}{*}{$H_{\mathrm{s}}$} & & Intercept & 0.520 & & 0.531 & \\
\hline & Village & Ndieumeul & Ref. & & Ref. & \\
\hline & & Diokhor & $-0.008(-0.025,0.010)$ & 0.381 & $-0.004(-0.022,0.014)$ & 0.668 \\
\hline & & Pakh & $0.020(0.003,0.038)$ & $0.025^{*}$ & $0.014(-0.004,0.033)$ & 0.118 \\
\hline & Gender & Female & Ref. & & Ref. & \\
\hline & & Male & $0.002(-0.007,0.012)$ & 0.631 & $0.004(-0.005,0.012)$ & 0.418 \\
\hline & Co-infection & Present & Ref. & & Ref. & \\
\hline & & Absent & $-0.007(-0.018,0.005)$ & 0.238 & $-0.003(-0.015,0.008)$ & 0.547 \\
\hline & Age & & $0.001(-0.00007,0.0017)$ & 0.072 & $0.0004(-0.0004,0.001)$ & 0.360 \\
\hline & EPG & & $-2.10^{-6}\left(-9.10^{-6}, 5.10^{-6}\right)$ & 0.532 & $-2.10^{-6}\left(-1.10^{-5}, 6.10^{-6}\right)$ & 0.587 \\
\hline \multirow[t]{10}{*}{ AR } & & Intercept & 1.53 & & 1.55 & \\
\hline & Village & Ndieumeul & Ref. & & Ref. & \\
\hline & & Diokhor & $-0.011(-0.059,0.036)$ & 0.640 & $-0.014(-0.083,0.055)$ & 0.690 \\
\hline & & Pakh & $0.018(-0.031,0.067)$ & 0.456 & $0.022(-0.049,0.093)$ & 0.534 \\
\hline & Gender & Female & Ref. & & Ref. & \\
\hline & & Male & $-0.009(-0.036,0.018)$ & 0.510 & $-0.121(-0.046,0.022)$ & 0.482 \\
\hline & Co-infection & Present & Ref. & & Ref. & \\
\hline & & Absent & $-0.014(-0.045,0.018)$ & 0.389 & $-0.014(-0.057,0.029)$ & 0.521 \\
\hline & Age & & $0.001(-0.002,0.003)$ & 0.653 & $-6.10^{-5}(-0.003,0.003)$ & 0.967 \\
\hline & EPG & & $-6.10^{-6}\left(-3.10^{-5}, 1.10^{-5}\right)$ & 0.505 & $-1.10^{-5}\left(-4.10^{-5}, 1.10^{-5}\right)$ & 0.364 \\
\hline
\end{tabular}

Abbreviations: AR, allelic richness; $\mathrm{Cl}$, confidence interval; EPG, eggs per gram of feces; $F_{\mathrm{IS}}$, inbreeding coefficient; $H_{0}$, observed heterozygosity; $H_{\mathrm{s}}$, unbiased expected heterozygosity; NA, not applicable.

Significance was indicated with * when $P<0.05$ and ** when $P<0.006$ (that is, Bonferroni corrected). 
Table 4 Jack-knifed estimates of Pearson's correlation tests between human host age on the one hand and $S$. mansoni inbreeding and heterozygosity on the other

\begin{tabular}{|c|c|c|c|c|}
\hline & \multicolumn{2}{|c|}{ Host age_-parasite $\mathrm{F}_{I S}$} & \multicolumn{2}{|c|}{ Host age_-parasite $\mathrm{H}_{o}$} \\
\hline & r & P-value & $r$ & P-value \\
\hline L46951 & -0.316 & $0018 *$ & 0.405 & $0005^{*}$ \\
\hline CA11-1 & -0.377 & $0004^{*}$ & 0.423 & $0,003 *$ \\
\hline S9-1 & -0.350 & $0008^{*}$ & 0.383 & $0008^{*}$ \\
\hline SMD11 & -0.347 & 0009* & 0.437 & $0002^{* *}$ \\
\hline SMD25 & -0.339 & $0011^{*}$ & 0.415 & $0004 *$ \\
\hline SMD28 & -0.357 & $0007^{*}$ & 0.385 & $0006 *$ \\
\hline SMD43 & -0.392 & $0003^{*}$ & 0.455 & $0001^{* *}$ \\
\hline SMD89 & -0.361 & $0006^{*}$ & 0.398 & $0006 *$ \\
\hline SMDA28 & -0.315 & $0018^{*}$ & 0.423 & $0001^{* *}$ \\
\hline Overall & -0.350 & $0008^{*}$ & 0.429 & $0001^{* *}$ \\
\hline
\end{tabular}

Abbreviations: $F_{\mathrm{IS}}$, inbreeding coefficient; $H_{0}$, observed heterozygosity.

Significance was indicated with * when $P<0.05$ and ** when $P<0.003$ (that is, Bonferroni corrected)

that were genotyped for $<13$ parasites (Table 3). AR did not reveal any significant effects (Table 3 ).

\section{DISCUSSION}

Schistosomiasis has a complex epidemiology with a substantial variation among human hosts in infection intensity and pathology. Within schistosomiasis endemic communities, infection intensities are related to host age, with an increase in intensity in early childhood, a peak in adolescence (8-15 years) and a decrease thereafter (Stelma et al., 1993; Meurs et al., 2012). This pattern could be explained by immunity that is acquired over age (Kabatereine et al., 1999), by differential water contact behavior with children being more exposed to infected water than adults (Fulford et al., 1996) and/or by other factors (for example, skin composition, hormones) that vary with age (Fulford et al., 1998). Here we found a negative correlation between human host age and $S$. mansoni inbreeding levels and a positive correlation between host age and parasite heterozygosity (Figure 3). These results indicate that older human hosts are to a greater extent infected by genetically unrelated parasites, resulting in heterozygous offspring. Such age-related differences could result from a number of mutually non-exclusive scenarios, which will be discussed below.

\section{Host immunity selects for unrelated $S$. mansoni parasites}

The concomitant immunity hypothesis states that adult schistosomes invoke a partial immunoprotective host response against new incoming larval schistosomes (Terry, 1994; Brown and Grenfell, 2001). This host protective immunity may be parasite genotypedependent, with the infectivity rate of incoming schistosomes being lower when genetically more similar to the (immunizing) adult schistosomes (Beltran et al., 2011). Such a genotype-dependent antigenicity could explain the observed decline in parasite inbreeding with host age: the first infecting schistosome genotypes are recognized by the host immune system that, upon super-infection, eliminate genetically similar genotypes more efficiently than unrelated schistosomes (Galvani, 2005). This would lead to an increased recruitment of genetically dissimilar worms with host age.

Hosts serve as genetic mixing bowls for S. mansoni parasites Given the long life expectancy of schistosome worms, older hosts may have acquired genetically unrelated parasites in space (if the action radius of the host increases with its age) and in time (if cercariae at a given transmission site are genetically dissimilar between time points). Human hosts then become 'genetic mixing bowls', where the accumulation of unrelated parasites during their lives results in an increase in the diversity of the respective parasite population (Curtis and Minchella, 2000).

On the other hand, children may be more exposed to related parasites than adults owing to differences in water contact behavior. Children in Senegal (Scott et al., 2003; Sow et al., 2011) and elsewhere (for example, Fulford et al., 1996) showed more and longer water contact than adults, and a study in Kenya showed that children visit fewer transmission sites than adults (Kloos et al., 1997). Children who visit the same transmission site frequently would have an increased risk of re-infection by $S$. mansoni strains that are related to the ones that previously contributed to the gene pool (that is, sib transmission; Anderson et al., 1995; Criscione et al., 2005). This will increase the chance for biparental inbreeding of $S$. mansoni parasites resulting in an increase in $F_{I S}$. If sib transmission were strong, however, most of the genetic variation would be found among (groups of) hosts (for example, Mulvey et al., 1991), while models have shown that gene flow of unrelated parasites and the mixing of larvae before or after asexual reproduction erode the effects of sib transmission (Prugnolle et al., 2005b). The present study revealed low genetic differentiation between $S$. mansoni parasite populations at the village and the host level, indicating high parasite gene mixing (Table 2). Furthermore, the mean pairwise relatedness between the cercariae from Ndieumeul did not differ significantly from its expected distribution, whereas the contrary would be expected if the pool consisted of siblings (Figure 2). Altogether, these results suggest that the impact of sib transmission on variations in $F_{\text {IS }}$ estimates within our study area (or at least within the transmission site of Ndieumeul) is probably minimal. This implies that the suggested role of age-dependent water contact behavior in schistosome inbreeding may be of little relevance within our study area. Preliminary data also showed that the majority of the inhabitants within our study area tend to visit the same transmission site regardless of their age, suggesting little differences in water contact behavior between age groups. It can, however, not be excluded that we missed water contact sites that are separated from the main transmission sites along Lake Guiers.

Similarly, a study on S. haematobium within two schools in Mali (Gower et al., 2011) found higher numbers of unique adult worms (as inferred from larval genotypes) in a school where the average age of the children was also higher than in the other school. A clear association between host age and parasite diversity was, however, not reported. This might be due to the fact that only parasites from children were sampled, which narrows the age range under study. However, a higher number of unique adult worms were found in the parasite populations recovered from boys in one of the two schools (Gower et al., 2011). A study in Zimbabwe also found higher levels of $S$. haematobium diversity in boys than in girls, and this was in accordance with the observation that males had more water contact and more intense infections than females (Brouwer et al., 2003). We, however, did not find any indication that gender would have an impact on the genetic constitution of $S$. mansoni infrapopulations (Table 3). An earlier epidemiological survey within the same villages found no differences between gender and risk of S. mansoni or S. haematobium infection or infection intensity (Meurs et al., 2012). Only older women showed higher risk for infection than older men, but the difference was not significant. Altogether, these results suggests that within our study area there might be little differences between gender or age in water contact or that gene flow is large 
a

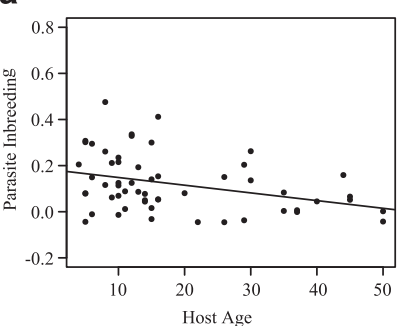

d

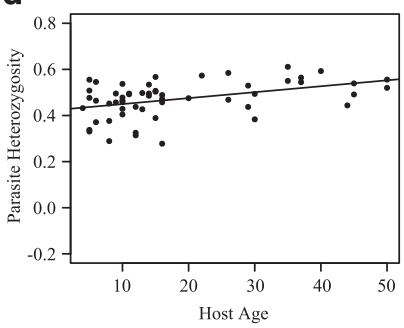

b

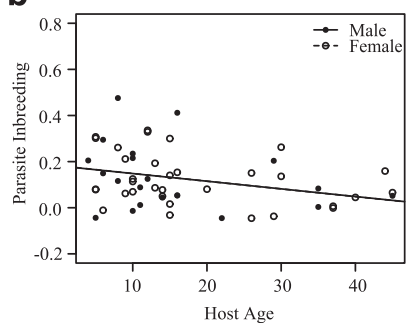

e

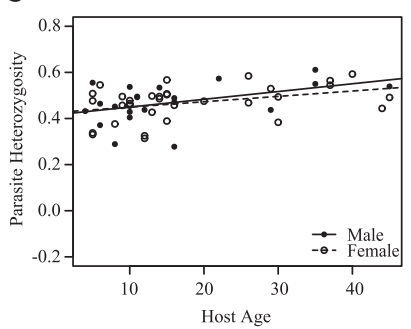

C

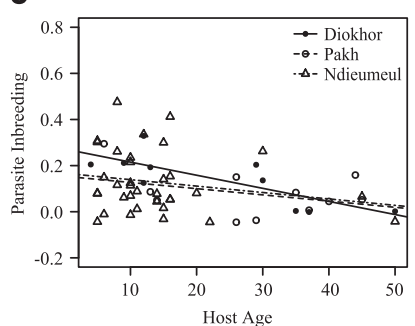

f

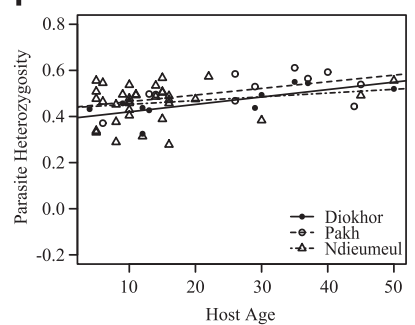

Figure 3 Linear regressions between human host age on the one hand and Schistosoma mansoni inbreeding (a-c) and heterozygosity (d-f) on the other. (b, e) According to host gender. (c, f) According to village.

enough to break down any association. Note that the fact that we reject the day-to-day water contact behavior as a potential explanation for higher levels of inbreeding within children does not necessarily reject the genetic mixing bowl hypothesis, as at a larger time scale older hosts could still have been exposed relatively more to unrelated parasites than children.

\section{Host mobility and Wahlund effect}

The fact that parasite genotypes are randomly distributed among hosts and villages (Table 2) may be explained by high host mobility. Previous studies hypothesized that the lack of spatial differentiation could be due to the absence of boundaries to water bodies, thereby facilitating host movement among transmission sites (Thiele et al., 2008; Agola et al., 2009; Steinauer et al., 2009). Indeed, the open and flat Sahel region within our study area may impose few restrictions on human host movement. In addition, all transmission sites are situated along (or near) the same water body (Lake Guiers), most likely facilitating both human and snail mobility among sites. Such a human host movement could result in a Wahlund effect when transmission sites represent spatially or temporally separated gene pools (that is, the presence of cryptic population structure). This Wahlund effect has often been used as an argument to explain positive deviations from HWE within natural schistosome populations (for example, Agola et al., 2006; Gower et al., 2011). Here, we showed that the impact of the Wahlund effect within our sampling area could be considered minimal owing to the absence of population structure and that positive deviations from HWE within natural schistosome populations could also result from non-random mating patterns that are linked with host-specific factors.

\section{CONCLUSIONS}

A significant association between host age on the one hand and parasite inbreeding and heterozygosity on the other was found. Our results show that young hosts are infected by related parasites, whereas there is an accumulation of unrelated strains as the host ages. We argue that these patterns could be explained by (1) genotype-dependent 'concomitant immunity', leading to selective recruitment of genetically unrelated worms with host age, and/or
(2) the 'genetic mixing bowl' hypothesis, where older hosts may have been exposed to a wider variety of schistosome strains than children. Regardless of the underlying mechanism, we have shown that host age may shape the genetic diversity of parasites and therefore their evolutionary potential. More field-based data are needed to confirm these results and to assess the relative contribution of each of these suggested mechanisms to the structuring of natural parasite populations.

\section{DATA ARCHIVING}

$\mathrm{R}$ scripts, parasite genotypes and human host-specific factors were deposited in the Dryad repository: doi:10.5061/dryad.6nm40. Note that the information on gender was excluded from the raw data to preserve anonymity.

\section{CONFLICT OF INTEREST}

The authors declare no conflict of interest.

\section{ACKNOWLEDGEMENTS}

We thank the villagers for their cooperation, the Senegalese field team (M Diop, A Fall, N Sy, M Wade, A Yague) for assisting with the fieldwork, Dr MHD Larmuseau for many discussions on population genetics, D Schaerlaekens for his comments on the manuscript and FA Kidane for her help with sampling and genotyping. FVdB is a doctoral fellow of the Flemish Interuniversity Council (VLADOC). TH is a postdoctoral fellow of the Research Foundation-Flanders (FWO-Vlaanderen). Research was benefited from a Research Grant of Research Foundation-Flanders (contract G.0552.10) and from travel grants awarded by the Flemish Interuniversity Council (VLIR) to FVdB and NB.

Agola LE, Mburu DN, DeJong RJ, Mungai BN, Muluvi GM, Njagi ENM et al. (2006). Microsatellite typing reveals strong genetic structure of Schistosoma mansoni from localities in Kenya. Infect Genet Evol 6: 484-490.

Agola LE, Steinauer ML, Mburu DN, Mungai BN, Mwangi IN, Magoma GN et al. (2009) Genetic diversity and population structure of Schistosoma mansoni within human infrapopulations in Mwea, central Kenya assessed by microsatellite markers. Acta Trop 111: 219-225. 
Anderson TJ, Romero-Abal ME, Jaenike J (1995). Mitochondrial DNA and Ascaris microepidemiology: the composition of parasite populations from individual hosts, families and villages. Parasitology 110: 221-229.

Belkhir K, Borsa P, Chikhi L, Raufaste N, Bonhomme F (1996-2004). GENETIX 4.05, logiciel sous Windows TM pour la génétique des populations. Laboratoire Génome, Populations, Interactions, CNRS UMR 5000, Université de Montpellier II, Montpellier (France).

Belkhir K, Castric V, Bonhomme F (2002). IDENTIX, a software to test for relatedness in a population using permutation methods. Mol Ecol Notes 2: 611-614.

Beltran S, Gourbal B, Boissier J, Duval D, Kieffer-Jaquinod S, Pierce RJ et al. (2011). Vertebrate host protective immunity drives genetic diversity and antigenic polymorphism in Schistosoma mansoni. J Evol Biol 24: 554-572.

Blair L, Webster JP, Barker GC (2001). Isolation and characterization of polymorphic microsatellite markers in Schistosoma mansoni from Africa. Mol Ecol Notes 1: 93-95.

Brouwer KC, Ndhlovu PD, Wagatsuma Y, Munatsi A, Shiff CJ (2003). Urinary tract pathology attributed to Schistosoma haematobium: Does parasite genetics play a role? Am J Trop Med Hyg 68: 456-462.

Brown SP, Grenfell BT (2001). An unlikely partnership: parasites, concomitant immunity and host defence. Proc $R$ Soc London Ser B Biol Sci 268: 2543-2549.

Bush AO, Fernandez Jc, Esch GW, Seed JR (2001). Parasitism: The Diversity and Ecology of Animal Parasites. Cambridge University Press: Cambridge, UK.

Bush AO, Lafferty KD, Lotz JM, Shostak AW (1997). Parasitology meets ecology on its own terms: Margolis et al revisited. J Parasitol 83: 575-583.

Caillaud D, Prugnolle F, Durand P, Theron A, de Meeus T (2006). Host sex and parasite genetic diversity. Microbes Infect 8: 2477-2483.

Castric V, Bernatchez L, Belkhir K, Bonhomme F (2002). Heterozygote deficiencies in small lacustrine populations of brook charr Salvelinus Fontinalis Mitchill (Pisces, Salmonidae): a test of alternative hypotheses. Heredity (Edinb) 89: 27-35.

Criscione CD (2008). Parasite co-structure: broad and local scale approaches. Parasite 15: $439-443$.

Criscione CD, Blouin MS (2004). Life cycles shape parasite evolution: comparative population genetics of salmon trematodes. Evolution 58: 198-202.

Criscione CD, Poulin R, Blouin MS (2005). Molecular ecology of parasites: elucidating ecological and microevolutionary processes. Mol Ecol 14: 2247-2257.

Curtis J, Minchella DJ (2000). Schistosome population genetic structure: when clumping worms is not just splitting hairs. Parasitol Today 16: 68-71.

Curtis J, Sorensen RE, Minchella DJ (2002). Schistosome genetic diversity: the implications of population structure as detected with microsatellite markers. Parasitology 125: S51-S59.

Curtis J, Sorensen RE, Page LK, Minchella DJ (2001). Microsatellite loci in the human blood fluke Schistosoma mansoni and their utility for other schistosome species. Mol Ecol Notes 1: 143-145.

de Meeus T, Goudet J (2007). A step-by-step tutorial to use HierFstat to analyse populations hierarchically structured at multiple levels. Infect Genet Evol 7: 731-735.

Durand P, Sire C, Theron A (2000). Isolation of microsatellite markers in the digenetic trematode Schistosoma mansoni from Guadeloupe island. Mol Ecol 9: 997-998.

Evanno G, Regnaut S, Goudet J (2005). Detecting the number of clusters of individuals using the software STRUCTURE: a simulation study. Mol Ecol 14: 2611-2620.

French MD, Churcher TS, Basanez MG, Norton AJ, Lwambo NJ, Webster JP (2012). Reductions in genetic diversity of Schistosoma mansoni populations under chemotherapeutic pressure: the effect of sampling approach and parasite population definition. Acta Trop 128: 196-205.

Fulford AJ, Ouma JH, Kariuki HC, Thiongo FW, Klumpp R, Kloos H et al. (1996). Water contact observations in Kenyan communities endemic for schistosomiasis: methodology and patterns of behaviour. Parasitology 113: 223-241.

Fulford AJ, Webster M, Ouma JH, Kimani G, Dunne DW (1998). Puberty and age-related changes in susceptibility to schistosome infection. Parasitol Today 14: 23-26.

Galvani AP (2005). Age-dependent epidemiological patterns and strain diversity in helminth parasites. J Parasitol 91: 24-30.

Goudet J (2005). HIERFSTAT, a package for $\mathrm{R}$ to compute and test hierarchical F-statistics. Mol Ecol Notes 5: 184-186.

Gower CM, Gabrielli AF, Sacko M, Dembele R, Golan R, Emery AM et al. (2011). Population genetics of Schistosoma haematobium: development of novel microsatellite markers and their application to schistosomiasis control in Mali. Parasitology 138: 978-994.
Gryseels B (1994). Human resistance to Schistosoma infections: age or experience? Parasitol Today 10: 380-384.

Hedrick PW (2005). A standardized genetic differentiation measure. Evolution 59: 1633-1638.

Kabatereine NB, Vennervald JB, Ouma JH, Kemijumbi J, Butterworth AE, Dunne DW et al. (1999). Adult resistance to schistosomiasis mansoni: age-dependence of reinfection remains constant in communities with diverse exposure patterns. Parasitology 118 . 101-105.

Kloos H, Fulford AJC, Butterworth AE, Sturrock RF, Ouma JH, Kariuki HC et al. (1997). Spatial patterns of human water contact and Schistosoma mansoni transmission and infection in four rural areas in Machakos District. Kenya Soc Sci Med 44: 949-968.

Meirmans PG (2006). Using the AMOVA framework to estimate a standardized genetic differentiation measure. Evolution 60: 2399-2402.

Meurs L, Mbow M, Vereecken K, Menten J, Mboup S, Polman K (2012). Epidemiology of mixed Schistosoma mansoni and Schistosoma haematobium infections in northern Senegal. Int J Parasitol 42: 305-311.

Mulvey M, Aho JM, Lydeard C, Leberg PL, Smith MH (1991). Comparative population genetic-structure of a parasite (Fascioloides-Magna) and its definitive host. Evolution (NY) 45: 1628-1640.

Nadler SA (1995). Microevolution and the genetic-structure of parasite populations. J Parasitol 81: 395-403.

Price PW (1980). Evolutionary biology of parasites. Monogr Popul Biol 15: 1-237.

Pritchard JK, Stephens M, Donnelly P (2000). Inference of population structure using multilocus genotype data. Genetics 155: 945-959.

Prugnolle F, Roze D, Theron A, DE Meeus T (2005b). F-statistics under alternation of sexual and asexual reproduction: a model and data from schistosomes (platyhelminth parasites). Mol Ecol 14: 1355-1365.

Prugnolle F, Theron A, Pointier JP, Jabbour-Zahab R, Jarne P, Durand P et al. (2005a). Dispersal in a parasitic worm and its two hosts: consequence for local adaptation. Evolution 59: 296-303.

Scott JT, Diakhate M, Vereecken K, Fall A, Diop M, Ly A et al. (2003). Human water contacts patterns in Schistosoma mansoni epidemic foci in northern Senegal change according to age, sex and place of residence, but are not related to intensity of infection. Trop Med Int Heal 8: 100-108.

Sire C, Durand P, Pointier JP, Theron A (2001). Genetic diversity of Schistosoma mansoni within and among individual hosts (Rattus rattus): infrapopulation differentiation at microspatial scale. Int J Parasitol 31: 1609-1616.

Sow S, de Vlas SJ, Stelma F, Vereecken K, Gryseels B, Polman K (2011). The contribution of water contact behavior to the high Schistosoma mansoni infection rates observed in the Senegal River Basin. BMC Infect Dis 11: 198.

Steinauer ML, Blouin MS, Criscione CD (2010). Applying evolutionary genetics to schistosome epidemiology. Infect Genet Evol 10: 433-443.

Steinauer ML, Christie MR, Blouin MS, Agola LE, Mwangi IN, Maina GM et al. (2013). Non-invasive sampling of schistosomes from humans requires correcting for family structure. PLoS Negl Trop Dis 7: e2456.

Steinauer ML, Hanelt B, Agola LE, Mkoji GM, Loker ES (2009). Genetic structure of Schstosoma mansoni in western Kenya: the effects of geography and host sharing. Int $J$ Parasitol 39: 1353-1362.

Stelma FF, Talla I, Polman K, Niang M, Sturrock RF, Deelder AM et al. (1993). Epidemiology of Schistosoma mansoni infection in a recently exposed community in northern Senegal. Am J Trop Med Hyg 49: 701-706.

Terry RJ (1994). Human immunity to schistosomes: concomitant immunity? Parasitol Today 10: 377-378.

Thiele EA, Sorensen RE, Gazzinelli A, Minchella DJ (2008). Genetic diversity and population structuring of Schistosoma mansoni in a Brazilian village. Int J Parasitol 38: 389-399.

Van den Broeck F, Geldof S, Polman K, Volckaert FAM, Huyse T (2011). Optimal sample storage and extraction procotols for reliable multilocus genotyping of the human parasite Schistosoma mansoni. Infect Genet Evol 11: 1413-1418.

Weir BS, Cockerham CC (1984). Estimating F-statistics for the analysis of populationstructure. Evolution (NY) 38: 1358-1370.

WHO (2006). Preventive Chemotherapy in Human Helminthiasis: Coordinated Use of Anthelminthic Drugs in Control Interventions: a Manual for Health Professionals and Programme Managers. World Health Organization: Geneva.

Supplementary Information accompanies this paper on Heredity website (http://www.nature.com/hdy) 